\title{
OTR3 - NEW APPROACHES FOR STANDARDIZATION AND VALIDATION OF QRT - PCR ASSAYS FOR QUANTITATION OF YELLOW FEVER ON CLINICAL SAMPLES WITH HIGH QUALITY PARAMETERS
}

Alice G. Fernandes $^{1,2}$, Gisela F. Trindade ${ }^{2}$, Anna M. Yoshida ${ }^{2}$, Constança Britto $^{1}$, Sheila Maria B. Lima².

1 Oswaldo Cruz Institute, Oswaldo Cruz Foundation, Rio de Janeiro, Brazil.

2 Bio-manguinhos, Oswaldo Cruz Foundation, Technological development, Rio de Janeiro, Brazil.

Objectives: The development and production of viral vaccines involves several steps that need the monitoring of viral load throughout the process (antigen production, purification, inactivation). Currently, these steps are monitored by plaque lysis titration assay, whose results take about seven to ten days to come out. With the advent of real time RT-PCR, we have a faster approach available to this issue. In this context, the development, standardization and validation of a technique to quickly and efficiently quantify the yellow fever (YF) virus in the aforementioned stages is extremely important.

Methods: To accomplish that, we constructed a plasmidial standard curve and validation parameters were evaluated. Furthermore, we defined the limits of detection and quantification of the test. To ensure high quality, internal controls were established in order to avoid false negative results.

Results: The statistical analysis revealed an excellent correlation between the results obtained in RNA copies/mL quantified by qRT-PCR and the viral titer calculated by lysis plaques tests $(\mathrm{R}=0.96)$. In addition, a correlation factor for conversion of the real time PCR data to plaque assays was generated. The results analysis showed that the validation experiments sufficed all parameters defined by the quality control sector.

Conclusion: The technique herein standardized proved to be effective for determining YF viral load both in vivo and in vitro, thus becoming a very important tool in all projects developed in LATEV, and may eventually be adopted as the gold standard laboratory analysis and quality control for vaccine production. 